\title{
Solar wind parameter and seasonal variation effects on the South Atlantic Anomaly using Tsyganenko Models
}

\author{
Kirolosse M. Girgis ${ }^{1,2^{*}}$, Tohru Hada ${ }^{3,4}$ and Shuichi Matsukiyo 3,4
}

\begin{abstract}
We studied the space weather effects on the South Atlantic Anomaly (SAA) magnetic response using Tsyganenko models. For the physical parameters characterizing the SAA, the study considered the minimum magnetic field, the location (longitude and latitude) of the SAA center, and the area of the SAA. Regarding the space weather parameters, we considered the solar wind dynamic pressure, the interplanetary magnetic field components, $B_{y I M F}$ and $B_{z I M F}$, the Dst index, and the geodipole tilting angle. To study the magnetic field response of the SAA, several different versions of the Tsyganenko models, namely, T96, T01, and TS05, were used to describe the external magnetic field contributions. The main internal magnetic field was calculated by the International Geomagnetic Reference Field (IGRF-12). The magnetic field study of the SAA was realized in long- and short-term (seasonal and diurnal) variations. We found that the Dst index and the geodipole tilting angle were the strongest influencing parameters on the SAA magnetic field response at all altitudes. Moreover, it was revealed that both magnetic poles might be a possible cause of the SAA magnetic field response, resulting from the space weather conditions. Furthermore, the magnetic field behavior of the SAA was affected by hourly variations, where the largest changes occurred at dayside.
\end{abstract}

Keywords: South Atlantic Anomaly, Tsyganenko models, Space weather

\section{Introduction}

The Earth's lowest magnetic field intensity is expanding from Africa, through South Atlantic Ocean to South America, hence the so-called South Atlantic Anomaly (SAA). Recently, this phenomenon is interpreted as the presence of negative geomagnetic fluxes at the coremantle boundary under the Earth's surface, which in turn decreases locally the field strength. Such an interpretation is discussed in detail by Terra-Nova et al. (2017), Tarduno et al. (2015), Cottaar and Lekic (2016) and Aubert (2015).

The SAA is an important subject of research in several scientific fields, such as in geomagnetism, where PavónCarrasco and De Santis (2016) studied the possible

\footnotetext{
*Correspondence: girgiskirolosse@esst.kyushu-u.ac.jp

${ }^{2}$ Aerospace Engineering Department, Faculty of Engineering, Cairo

University, Giza, Egypt

Full list of author information is available at the end of the article
}

geomagnetic reversal, in space plasma physics, to understand the close approach of the inner radiation belt, and in spacecraft design, to mitigate the radiation effects on the on-board instrument at low earth orbit (LEO), etc. In this paper, we are addressing our efforts to study the space weather effects on the magnetic field response of the South Atlantic Anomaly.

Because space weather activity is directly affecting the trapped radiation belts and the inner magnetosphere, much research had been carried out in studying the effects of space weather on the South Atlantic Anomaly. For example, the SAA magnetic field long-term response of the center of its location, due to proposed sinusoidal profiles of the solar wind ram pressure, the $\mathrm{z}$-component of the interplanetary magnetic field, $B_{z I M F}$, and the Dst index, using Tsyganenko model (T96), was researched by Qin et al. (2014), as well as Schaefer et al. (2016) and Ye et al. (2017). Moreover, the effect of the Dst index on the short-term response of the SAA proton maximum 
flux and area, using NOAA 17 data, was studied by Zou et al. (2015). In both studies, the results were obtained for specific and fixed altitudes, and for two parameters each. Furthermore, in this study's previous paper (Girgis and Hada 2018), solar wind ram pressure and IMF components on the SAA's magnetic field response using Tsyganenko model T96 were studied, by maintaining a constant Dst index. In addition, the solar cycle variation effects on the SAA proton flux were also studied by Nakano and Heckman (1968) and Dragt (1971), on its westward drift rate by Grigoryan et al. (2008), on its northward drift rate by Badhwar (1997) and Casadio and Arino (2011), as well as the anti-correlation between the solar radio flux $F_{10.7}$ and the proton flux by Huston et al. (1996) and Huston and Pfitzer (1998).

Since the implementation of the Tsyganenko models is the core of our study, we should confirm their accuracy at low altitudes. First of all, we recall that the net external magnetic field is described as a superposition of several magnetospheric currents: the large-scale field-aligned current systems, the magnetopause currents, the tail current sheet, and the ring current, as already described in great detail by the review article (Tsyganenko 2013), whose author presented his large contribution for conceiving the data-based models of the Earth's dynamic magnetosphere. Second, one of the methods to investigate such accuracy is realized by calculating the isotropic precipitation boundary (IB) from the current models and compare the results with the observed values deduced from low-altitude spacecraft measurements: Sergeev and Tsyganenko (1982) compared the computed adiabatic drift shells from T79 model (Tsyganenko 1979) for particles mirroring at $400 \mathrm{~km}$ with the experimental data, and found a good agreement with an error of $1^{\circ}$ at high latitudes. Weiss et al. (1997) compared the modeled IB from T89 (Tsyganenko 1989) with geosynchronous spacecraft and the low-altitude DMSP spacecraft $(830 \mathrm{~km})$ data and estimated the error of the median latitudinal difference to be about $2^{\circ}$. In addition, Shevchenko et al. (2010) calculated the IB by T96 model (Tsyganenko 1996) and compared it with THEMIS data at ionospheric altitudes and found a reasonable agreement with an error of about $1^{\circ}$ in latitude. Kubyshkina et al. (2009) found an error of about $1^{\circ}$ in quiet conditions using the same T96 model, compared with NOAA spacecraft, which can reach several degrees during substorm time. Ganushkina et al. (2005) estimated the maximum error to be about $0.7^{\circ}$, when $D s t=-16 \mathrm{nT}$, by implementing T01 model. Glancing at the ranges of the Dst index, the model under-estimates the tail current, where this issue was improved in TS05 model. Third, another method to test the accuracy of Tsyganenko models at low-altitudes can be achieved by calculating the geomagnetic cutoff latitude and comparing it with spacecraft measurements, as shown by Smart and Shea (2005), where the authors implemented T89 model with IGRF, and compared the obtained results with SAMPEX spacecraft data at $450 \mathrm{~km}$. The authors concluded that the modeled and the measured cutoff latitudes exhibit the same general trend, and during magnetic active periods, the estimated error was slightly increased. Further studies on this subject could be found in Smart and Shea (1994) and Kress et al. (2015). Beside the accuracy of the models, a main advantage is their parametrization by the solar wind drivers and/or groundbased indices. Such merit allows to interpret the magnetospheric configuration related to quiet conditions and storm events. Generally speaking, since there is no perfect model that could fulfill all the user requirements, we concentrate our efforts in this study to understand quantitatively and statistically the general behavior of the SAA, due to the variations in solar wind parameters and seasonal changes.

In this paper, we extend the investigation of space weather influence on the SAA magnetic field response, by studying its correspondence with altitude and by introducing the following variables: (1) the area of the SAA calculated beyond a selected threshold of the magnetic field strength; (2) the minimum magnetic field at the center of the SAA $\left(B_{\min }\right)$; and (3) the location (latitude and longitude) of the SAA defined as a point where the magnetic field is minimized. Furthermore, the space weather parameters include two components of the Interplanetary Magnetic Field (IMF), $B_{y I M F}$ and $B_{z I M F}$, as well as three velocity components of the solar wind, namely, the solar wind ram pressure, the Dst index, and the geodipole tilting angle $(\mu)$.

The methodology applied in this research was realized by adopting several different versions of Tsyganenko models, T96, (Tsyganenko 1996), T01, (Tsyganenko 2002a, b), and TS05, (Tsyganenko and Sitnov 2005). This was to describe the external magnetic field by comparing the output results from the three models, besides implementing the IGRF-12 (International Geomagnetic Reference Field) to describe the internal (main) magnetic field.

\section{Materials and methods}

The SAA tends to attract energetic particles from the inner radiation belt, due to the reduced magnetic field strength. Thus, the magnetic field is an essential factor that can affect the particle flux response in this region. Therefore, the objective of this investigation is to evaluate, quantitatively, which space weather parameters would affect the SAA variables.

To realize this, we, first of all, demonstrated the SAA variables, as shown in Fig. 1a the minimum magnetic field (= SAA center), the area of the SAA, which 


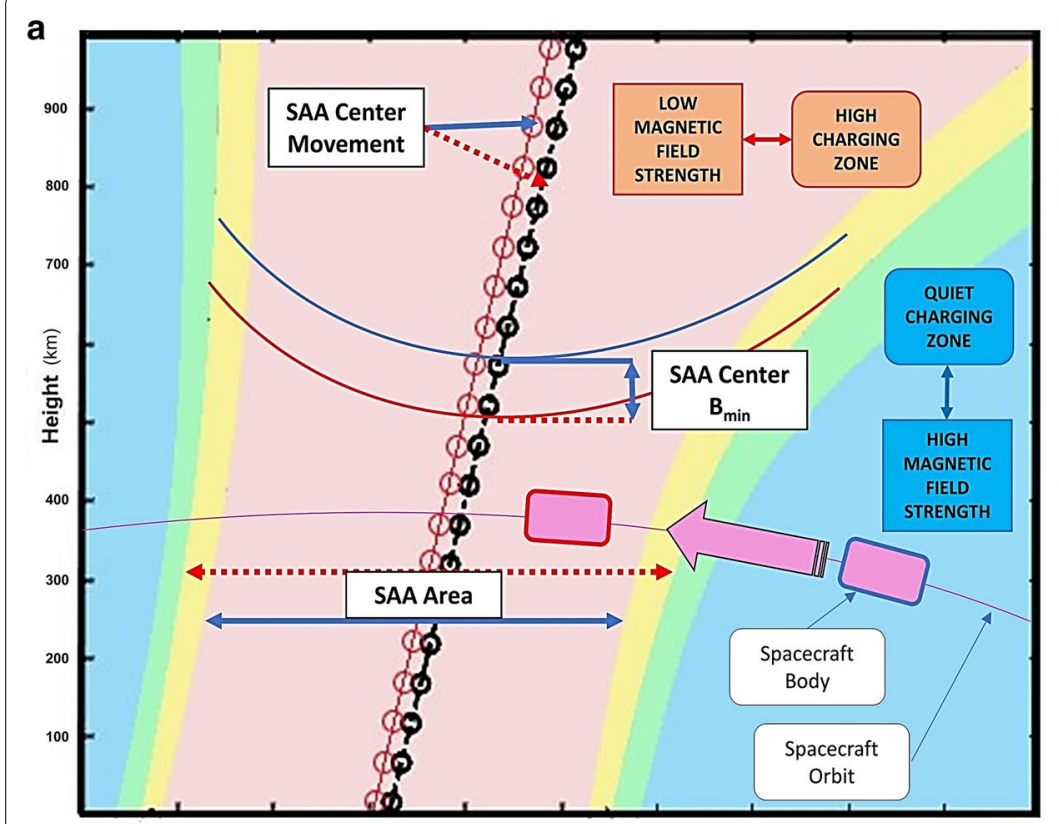

Longitude/Latitude (deg.)

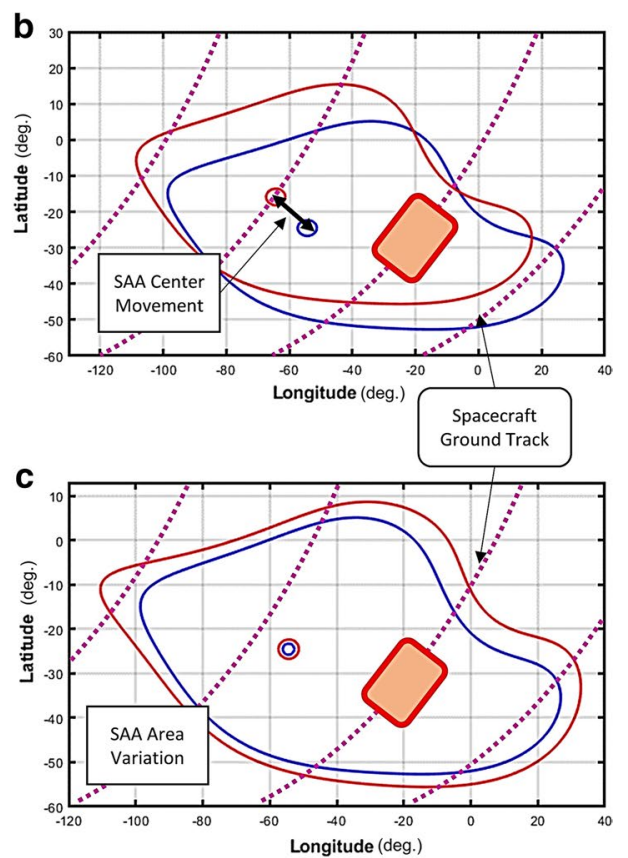

Fig. 1 SAA Parameters' Illustration. The SAA variables are displayed in two different views. The contours in a are colored based on the energetic particle (= radiation) population (light red for the high population and light blue for the low population). $\mathbf{b}, \mathbf{c}$ roughly demonstrate the movement and the expansion of the SAA, respectively

was calculated below the magnetic field threshold $\left(=7 / 6 \approx 1.167 B_{\min }\right)$, and the movement of the SAA center (= variations in longitude and latitude of the SAA center).

In addition, the study placed a typical spacecraft at Low Earth Orbit (LEO) (colored in magenta) that traveled across the SAA anomaly; a dangerous situation arose when the spacecraft body itself (rectangular shape colored with magenta) entered the high charging (= radiation) zone (colored in light red), whereas the safer region was outside of the SAA (colored in light blue). The movement of the SAA center location and the variation of the SAA area are shown in Panels (b) and (c), respectively, in addition to a typical ground track of a LEO satellite. It must be noted that the plot of both variations (red and blue contour lines) in all of the three panels was drawn for illustrative purposes, not according to realistic situations.

\section{SAA variables}

The variations of the SAA variables, as demonstrated in this study, were due to external magnetic field changes. They were calculated by subtracting their corresponding values, computed first, by adopting the internal (main) magnetic field only, as described by the IGRF model; and second, by adding the external magnetic field when defined by the Tsyganenko model, besides the internal (main) magnetic field. Normalization was then realized by dividing the resultant value by the variables, computed based on the internal (main) magnetic field, as shown in the simple following equations.

$$
\begin{aligned}
& \Delta\left[\bar{X}_{j}\right]_{\%}=\frac{\left[X_{j}\right]_{e+i}-\left[X_{j}\right]_{i}}{\left[X_{j}\right]_{i}} \times 100 \\
& {\left[X_{j}\right]=\left[\begin{array}{c}
A \\
B_{\min } \\
\theta \\
\phi
\end{array}\right]}
\end{aligned}
$$

where $X_{j}$ is a vector of the four SAA variables $(j=4)$; $A$ is the area; $B_{\min }$ is the minimum magnetic field intensity (= SAA center); $\theta$ is the latitude of the SAA center; $\phi$ is the longitude of the SAA center; $[X]_{e+i}$ is the SAA variable computed based on the external and the internal magnetic field, and; $[X]_{i}$ is the SAA variable computed based on internal magnetic field only.

\section{Input data}

The required input data to run Tsyganenko model are real solar wind data, such as the three velocity components of the solar wind, namely, the ram pressure, and the IMF components, $B_{y I M F}$ and $B_{z I M F}$. The daily data were provided by the ACE spacecraft from the years 2010 to 
2014, in addition to the Dst index and the geodipole tilting angle $(\mu)$, computed by the GEOPACK package subroutines. All of the information is plotted as shown in Fig. 2. It was expected that the changes in the input space weather variables not only would affect the global magnetic field configuration but would also evoke variations in the inner magnetosphere. Since the global structure of the Earth's magnetic field was calculated dependent on all of the implemented space weather parameters, a statistical study was carried out, to study the correlation between each of the space weather parameters and the SAA variables, besides demonstrating their temporal evolution, as shown in the next section.

\section{Results}

Figure 3 demonstrates the temporal evolution of the two SAA variables: the variation of the area of the SAA and that of the $B_{\min }$ plotted in Panels (a) and (b), respectively. The calculations were made for an altitude of $800 \mathrm{~km}$, using three Tsyganenko models T96, T01 and TS05.

At first glance, the study found that the variations in all of the SAA variables were larger when models T96 and T01 were used, compared with the runs where TS05 was used. In addition, the TS05 results clearly demonstrated a smoother sinusoidal profile, from which it was understood about the strong influence of the geodipole tilting angle on the SAA magnetic response.

The study further interpreted these results quantitatively regarding the relation between the SAA response
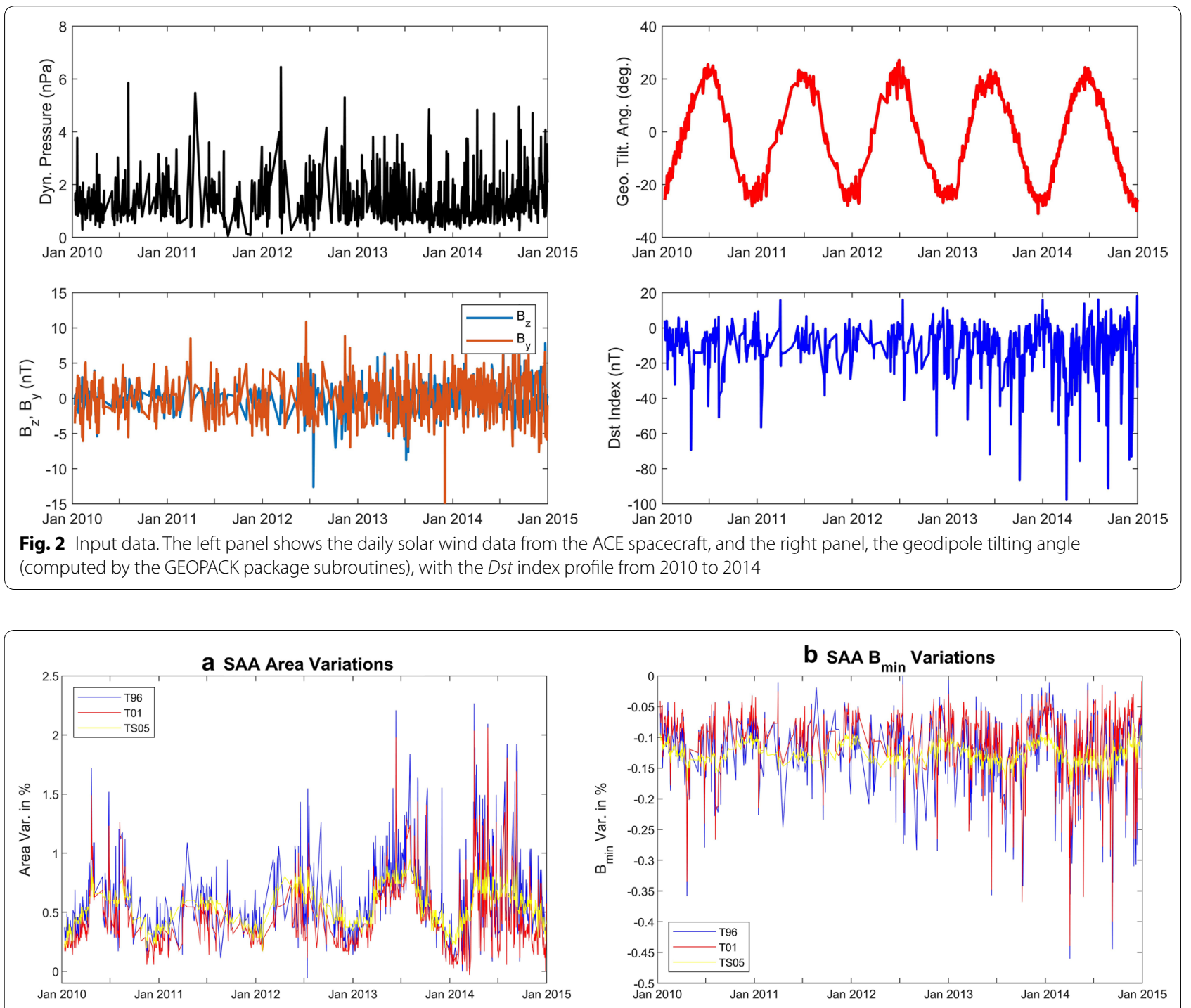

Fig. 3 Temporal variations of the two SAA variables. a represents the area and $\mathbf{b}$ the $B_{\mathrm{min}}$, by adopting the Tsyganenko models T96, T01, and TS05, at an altitude of $800 \mathrm{~km}$ 
according to the space weather conditions by performing a statistical study, which indicated a degree of correlation between each variation of the SAA variables and the space weather parameters. The well-known Pearson correlation coefficient had been calculated for two vectors, one standing for every temporal variation of the SAA variables, as discussed earlier in Eqs. 1, 2; and the other one, for every temporal variation of the implemented space weather parameter, corresponding to a selected altitude, and calculated based on each Tsyganenko model, T96, T01 and TS05.

Figures 4, 5 display the correlation coefficient for every SAA variable, with respect to every one of the space weather parameters: the solar wind dynamic pressure, $B_{y I M F}, B_{z I M F}$, the geodipole tilting angle, and the Dst index, at altitudes of 100, 400, 700 and $1000 \mathrm{~km}$, using every Tsyganenko model, T96, T01 and TS05. It was found from the T96 calculations that the solar wind dynamic pressure was affecting all of the SAA variables, approximately at all altitudes. At a glance, the IMF had a very weak influence on all of the SAA variables, regardless of the Tsyganenko model used. However, there was still a moderate absolute correlation $(\approx 0.4)$ between $B_{y I M F}$ and SAA $B_{\min }$ when TS05 was used. Indeed, the three models agreed that both the geodipole tilting angle and the Dst index effects were dominant for all altitudes and for all of the SAA variables. Tsyganenko models T96 and T01 both emphasized the effects of the Dst index on all of the SAA variables; on the other hand, the TS05 calculations decreased the Dst index effect on the SAA behavior.

Table 1 summarizes the correlation analysis, as shown in Figs. 4 and 5, as obtained by the three Tsyganenko models, T96, T01 and TS05 at an altitude of $800 \mathrm{~km}$. The colored values in the table identify the weak (blue, $0.1-0.4$ ), moderate (green, $0.4-0.7$ ), and strong (red, $0.7-1)$ absolute correlation. It was again deduced numerically that the three models T96, T01 and TS05 agreed together that the Dst index and the geodipole tilting angle $(\mu)$ were the most influencing space weather parameters on the SAA magnetic response. Moreover, the longitudinal movement of the SAA center was the SAA variable that was least influenced by the external space weather conditions. In this study, since it has focused on the inner magnetosphere dynamics due to the solar storm conditions, it is recommended to take into account the calculations of both models: T01, which better resolves the inner magnetosphere and magnetotail structures, in addition to TS05, that modulates the inner magnetosphere's response, corresponding to the strong storm events.

\section{Discussion}

\section{Comparison of the results using Tsyganenko models T96, T01 and TS05}

When adopting T01 and TS05, the SAA variables became less affected by the solar wind ram pressure and the IMF components, in comparison to T96, which was more affected by the Dst index and the geodipole tilting angle. This meant that the magnetotail structure was playing an important role in the SAA magnetic field response, and hence, the inner magnetosphere. This result was also confirmed by Kronberg et al. (2015), who found that an acceleration of the ions in the inner radiation belts was strongly associated to the AE index and less to the changes in the IMF components and the solar wind ram pressure. The magnetotail dynamics were influential on the SAA magnetic response and this is clarified in the next section.

To interpret these results, it is essential to understand the differences between the three Tsyganenko models. As explained in Tsyganenko (1996), T96 was built to study the effects of space weather on the global magnetosphere configuration. T01 and TS05 were then developed to better define the inner magnetosphere response when associated with the space weather conditions (Tsyganenko 2002a, b; Tsyganenko and Sitnov 2005). One of the main differences between TS05 and T01 was the inclusion of different temporal responses of the individual geomagnetic field sources in the TS05 model. Therefore, Tsyganenko and Sitnov (2005) explicitly confirmed that TS05 was more accurate and physically consistent, since both the spatial configuration and the time evolution would become equally significant for describing the storm-time field.

\section{About the $D$ st index and the geodipole tilting angle effects on the SAA: a direct relationship with the magnetic pole variations}

From the previous analysis, it was found that the geodipole tilting angle and the Dst index were the most influencing space weather parameters on the SAA magnetic field response. The effects of both parameters on the global geomagnetic activities were also discussed in great detail by Malin and Isikara (1976) as well as Shore et al. (2016). This was where it was explained that the ring current moves latitudinally through the year, due to the tilting of Earth's rotational axis with respect to the ecliptic plane, so that the solar wind compression of the magnetosphere pushes the ring current toward the south during the Northern Hemisphere summer, and toward the north 6 months later.

Since the SAA is unlikely to be an isolated feature from the global Earth's magnetic field, the magnetic poles may "drive" the SAA response. Accordingly, it was further 


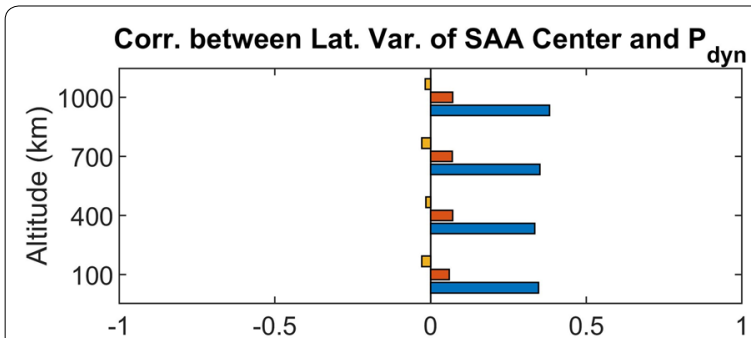

Corr. between Lat. Var. of SAA Center and B

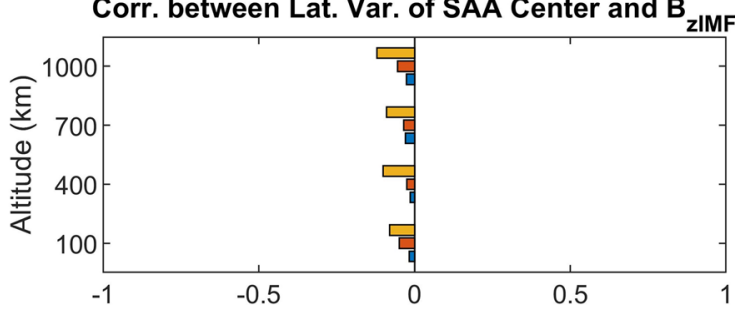

Corr. between Lat. Var. of SAA Center and B

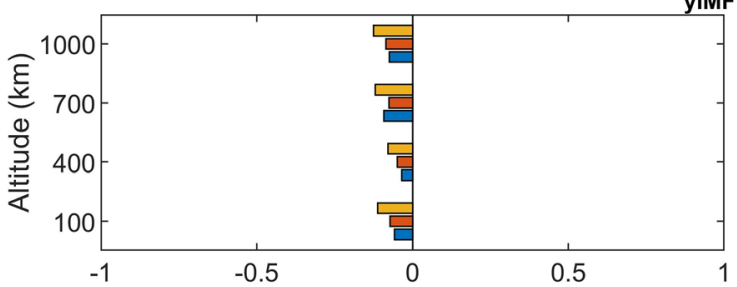

Corr. between Long. Var. of SAA Center and $P_{\text {dyn }}$

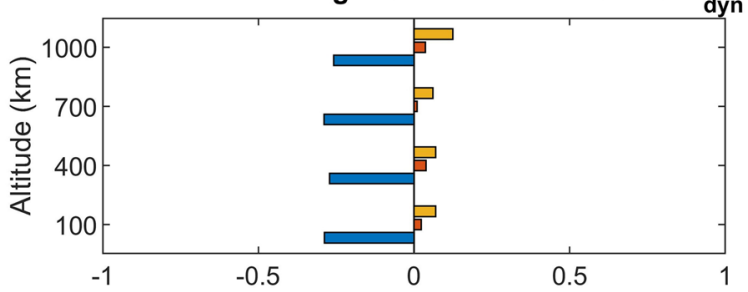

Corr. between Long. Var. of SAA Center and $B_{\text {zIMF }}$

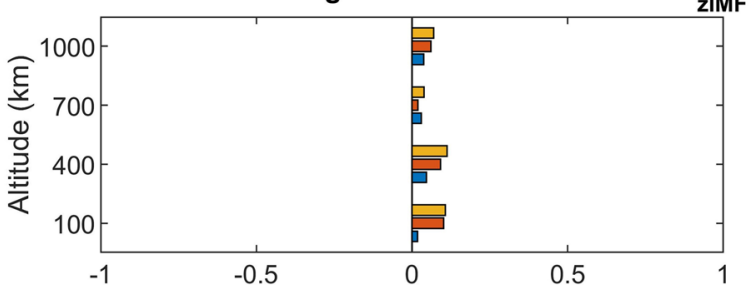

Corr. between Long. Var. of SAA Center and B

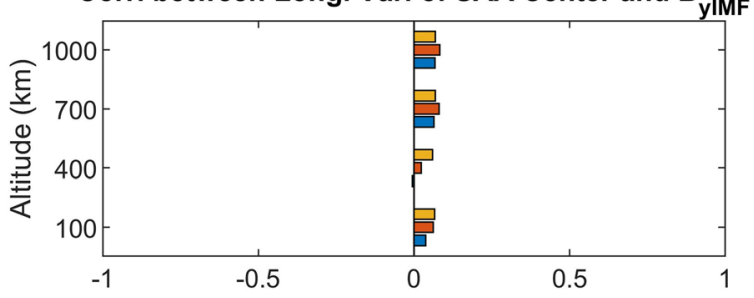

Corr. between Lat. Var. of SAA Center and Geod. Tilt Angle Corr. between Long. Var. of SAA Center and Geod. Tilt Angle
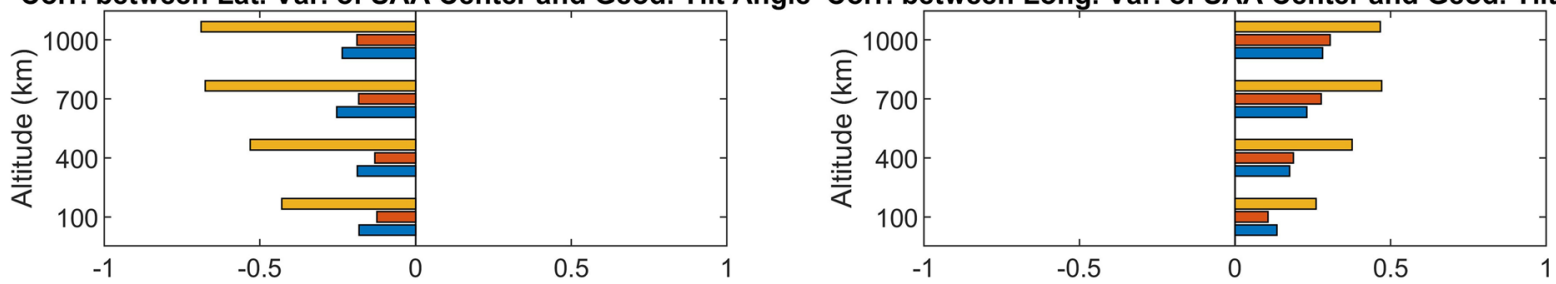

Corr. between Lat. Var. of SAA Center and |Dst| index
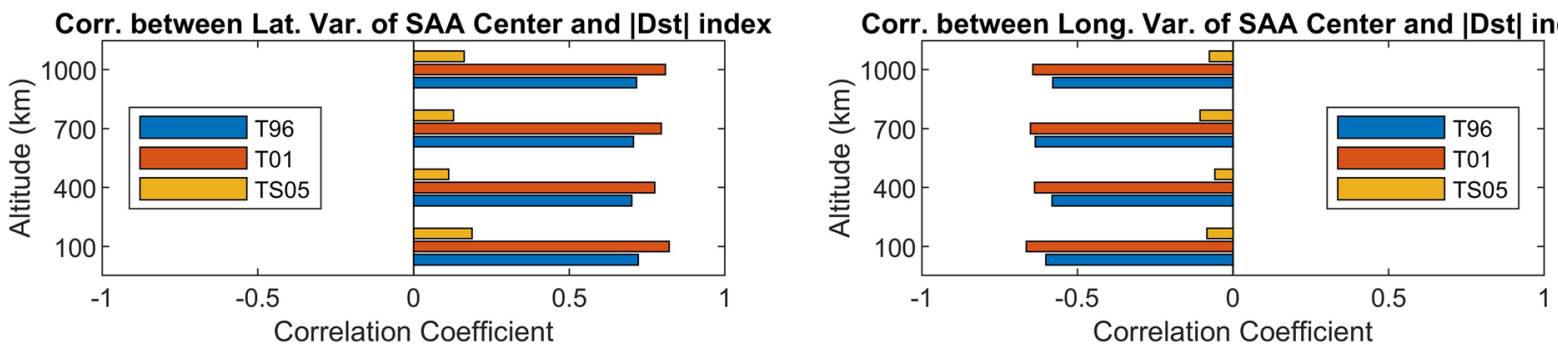

Fig. 4 Statistical study results. The statistical study illustrates the correlation coefficients of the latitudinal and longitudinal SAA center movements according to altitude for the Tsyganenko models, T96, T01 and TS05, referencing the different space weather parameters

studied regarding the issues of both of the magnetic poles' response to the space weather condition. Basically, the variables were the maximum magnetic field $B_{\max }$, the location (latitude and longitude) where the magnetic field was maximized, and the areas of the magnetic poles, defined as the region where the magnetic field was larger than $6 / 7(\approx 0.88)$ of $B_{\max }$. We have used the same data series as shown in Fig. 2.

From the study of both magnetic pole temporal variations, many interesting features were found: first, the magnetic poles are also precisely affected by the space weather parameters, namely, the geodipole tilting angle 

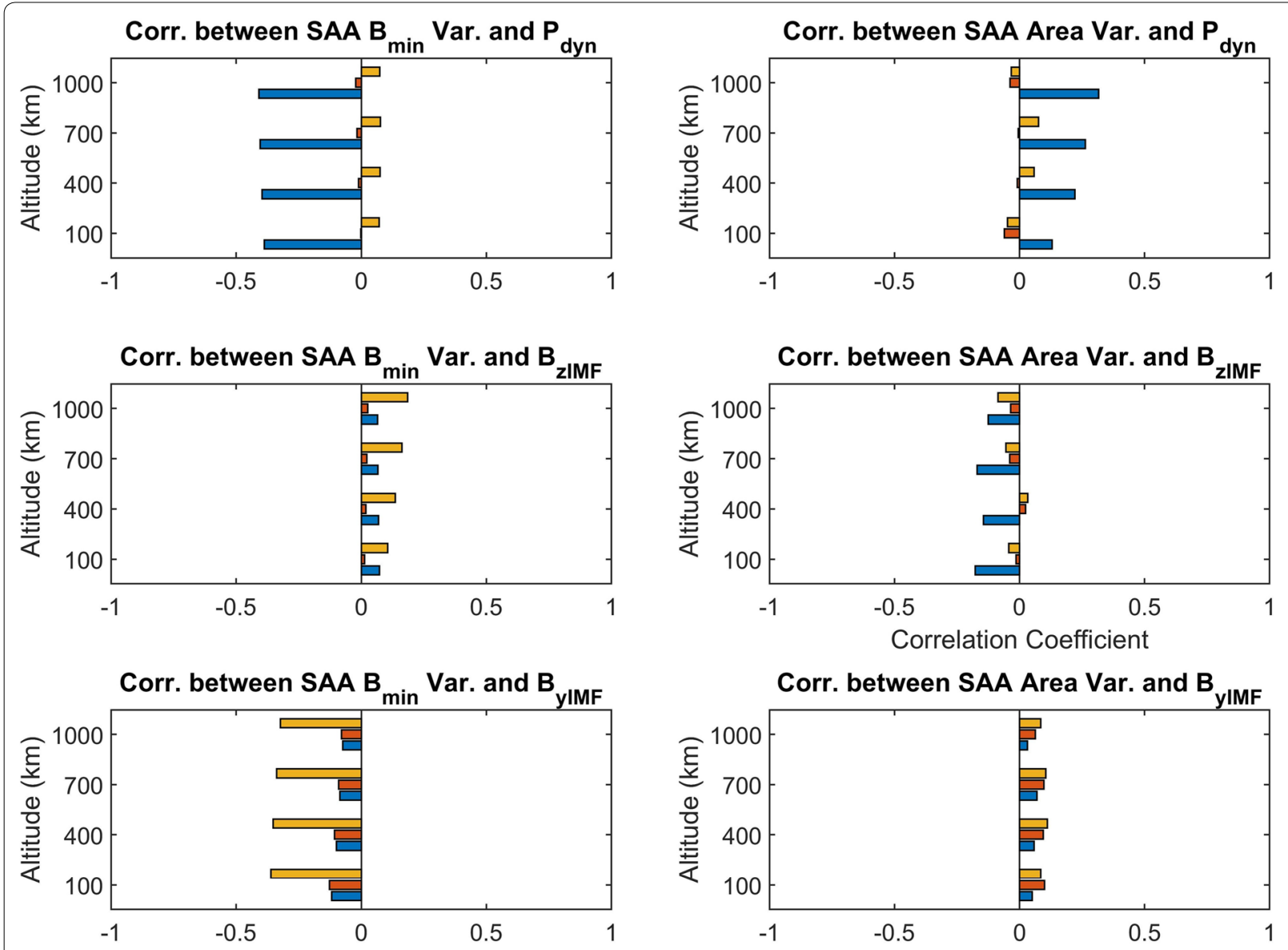

Corr. between SAA $B_{\text {min }}$ Var. and Geod. Tilt Angle

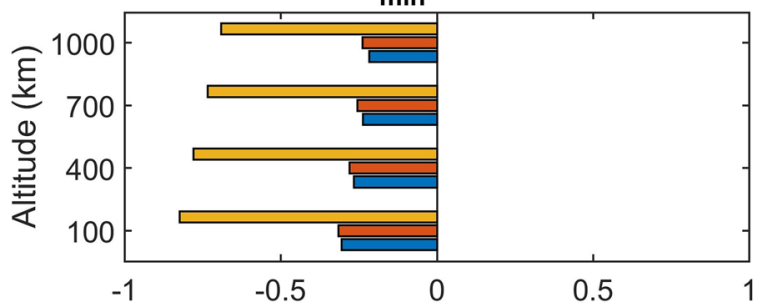

Corr. between SAA Area Var. and Geod. Tilt Angle

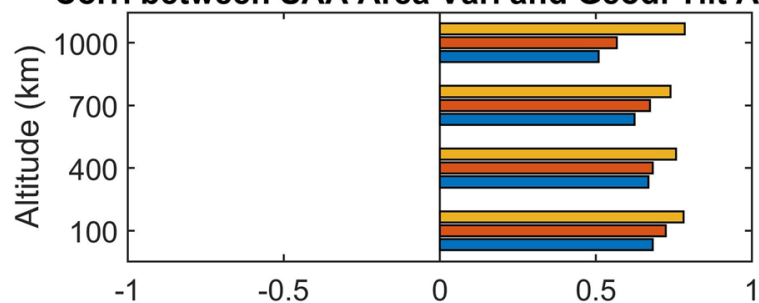

Corr. between SAA B ${ }_{\text {min }}$ Var. and [Dst| index

Corr. between SAA Area Var. and |Dst| index
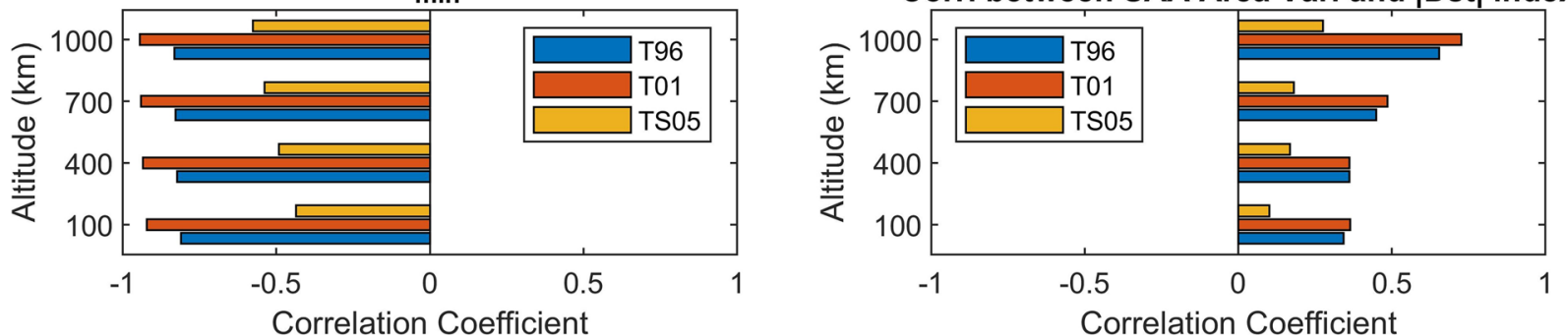

Fig. 5 Statistical study results. The statistical study illustrates the correlation coefficients of the area and the $B_{\min }$ of the SAA center according to altitude for the Tsyganenko models, T96, T01 and TS05, referencing the different space weather parameters 

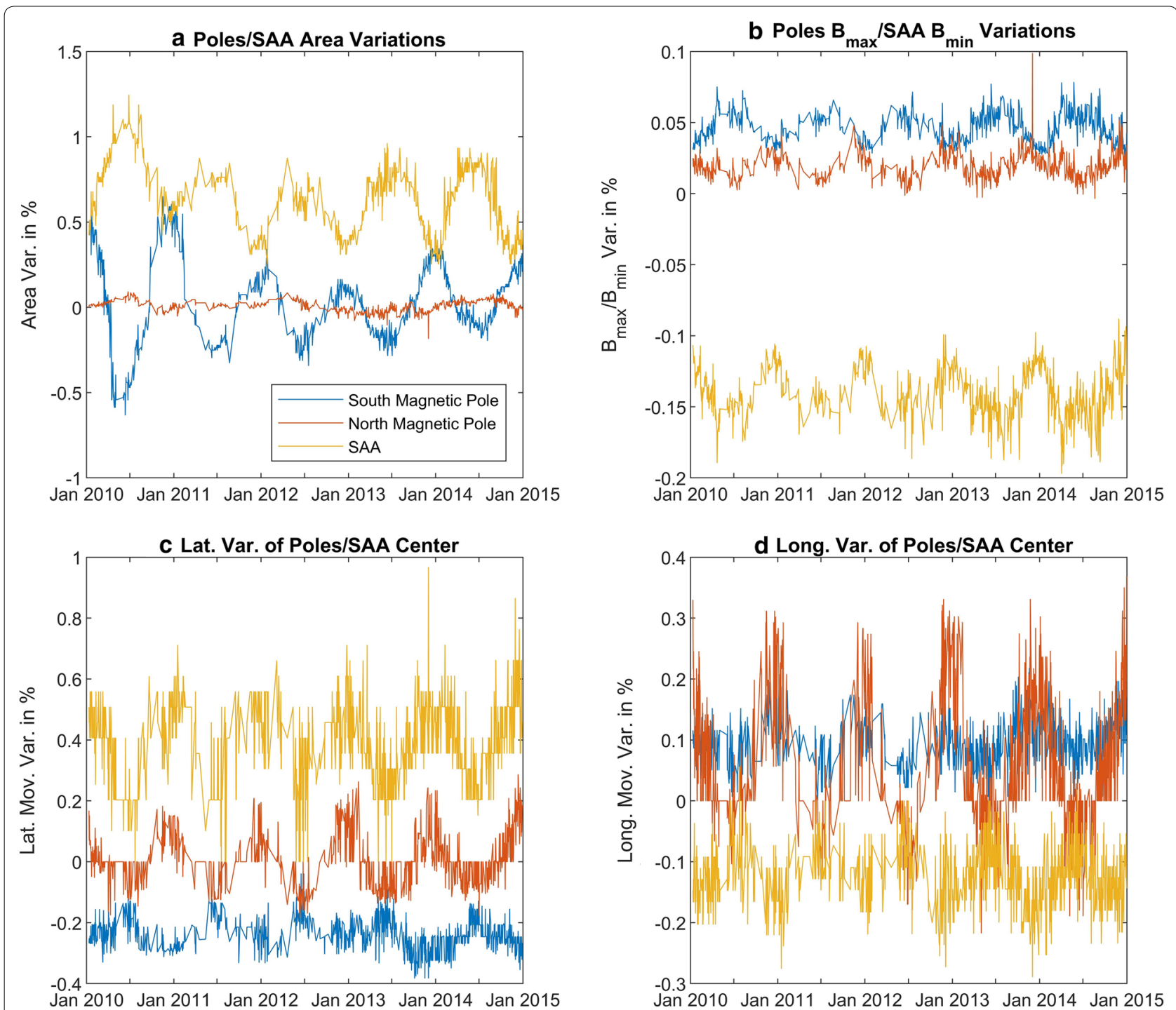

Fig. 6 Magnetic poles temporal variations. Magnetic poles and the SAA variation profiles for the four variables: the area, the minimum/maximum magnetic field intensity, the latitudinal and longitudinal center movements at $1000 \mathrm{~km}$ altitude

and the Dst index, as shown in Fig. 6. This figure demonstrates the temporal variations of both magnetic pole variables at an altitude of $1000 \mathrm{~km}$, as well as the correlation coefficients between the variations of the magnetic pole variables and the space weather parameters, at three different altitudes, 100, 500 and $1000 \mathrm{~km}$, as illustrated in Fig. 7. Second, it is easier to understand the relationship between both of the variables of the area, and the maximum magnetic field strength variations. From Figs. 6 and 7 , a global feature can be observed: the area variations are anti-correlated with the magnetic field changes, not only for the magnetic poles but also for the SAA, as shown earlier in the previous section.

\section{Geodipole tilting angle effects on the magnetic poles} and the $S A A$

Figure 8 demonstrates the geodipole tilting angle effects on both magnetic poles. Panels (a) and (b) show the magnetic field contour lines (in black solid lines) and the magnetic field lines (in white lines) for the positive and the negative geodipole tilting angles, $29.5^{\circ}$ and $-29.5^{\circ}$ respectively. Panels (c) and (d) are typically projected contour plots of the magnetic field in geodetic latitude and longitude terms. The white lines are the initial magnetic pole boundaries, and the black dotted lines are the boundaries that include the negative and positive geodipole tilting angle effects, as shown in Panels (c) and (d), respectively. Since the magnetic field variations cannot 

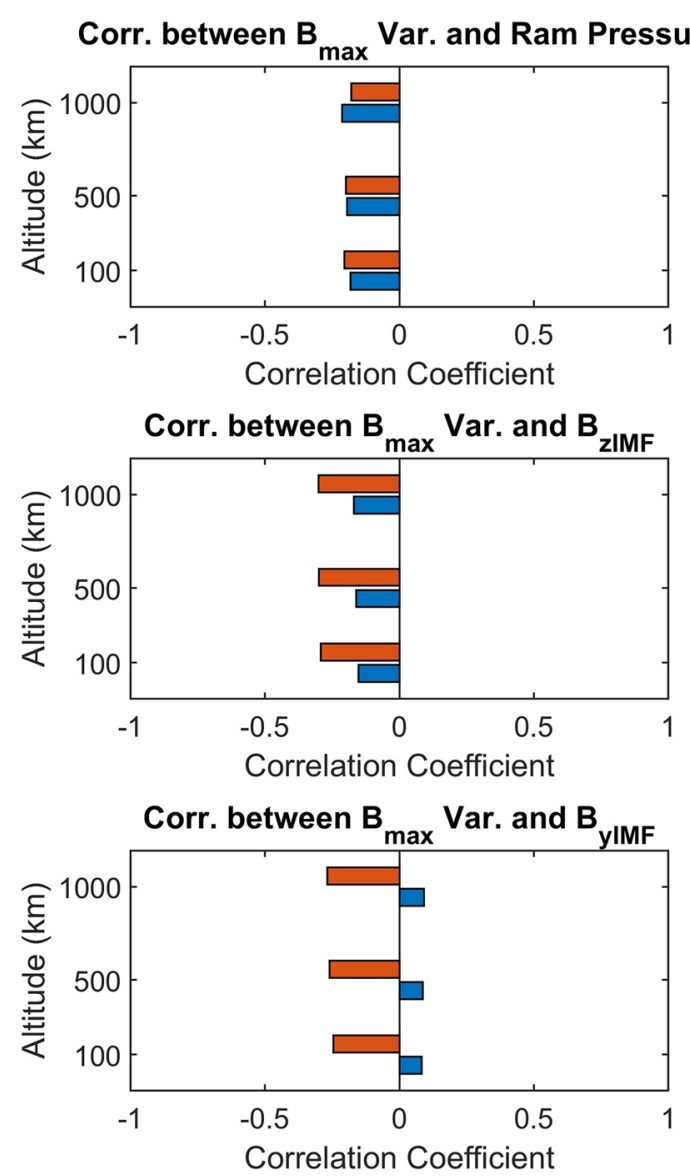

Corr. between $B_{\max }$ Var. and Geod. Tilt angle
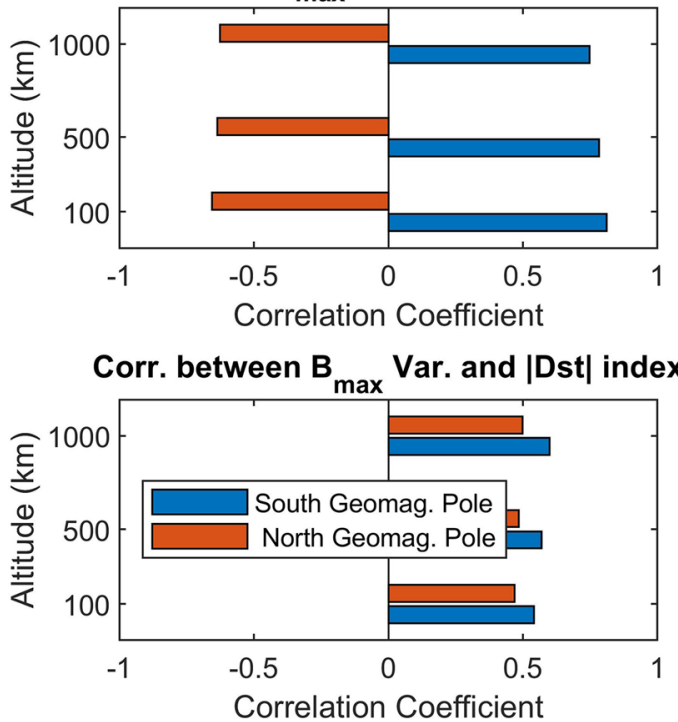

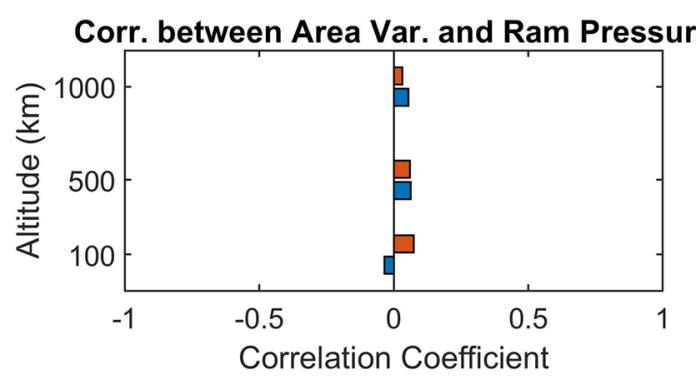

Corr. between Area Var. and $B$

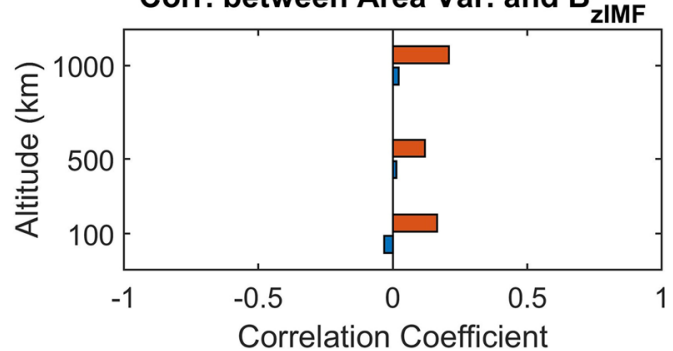

Corr. between Area Var. and B

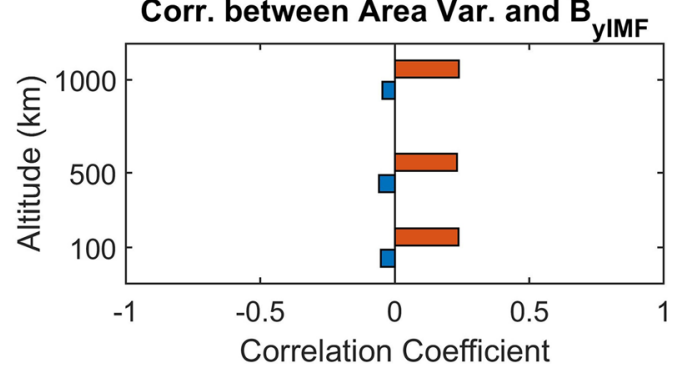

Corr. between Area Var. and Geod. Tilt angle

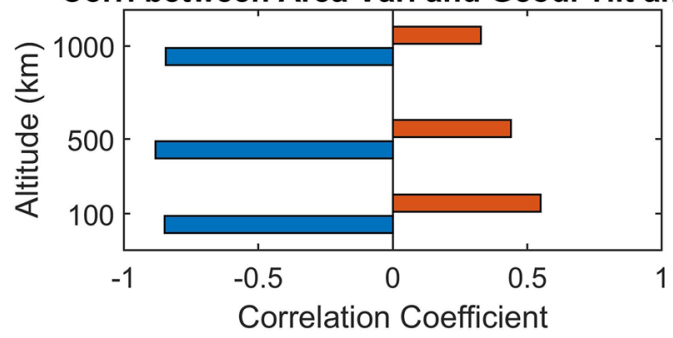

Corr. between Area Var. and [Dst| index

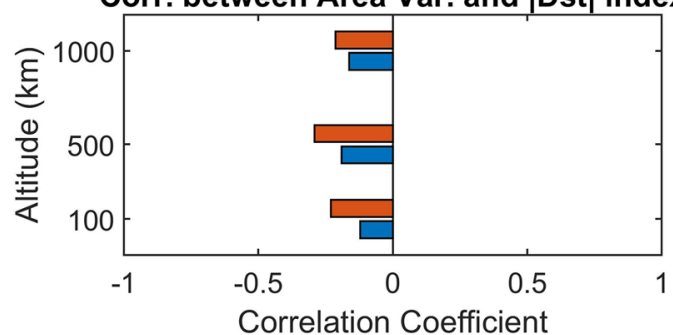

Fig. 7 Statistical study results. Correlation coefficients between the magnetic pole area and $B_{\text {max }}$ variations, with respect to the space weather parameters, and according to the altitudes, 100, 500, and $1000 \mathrm{~km}$ 


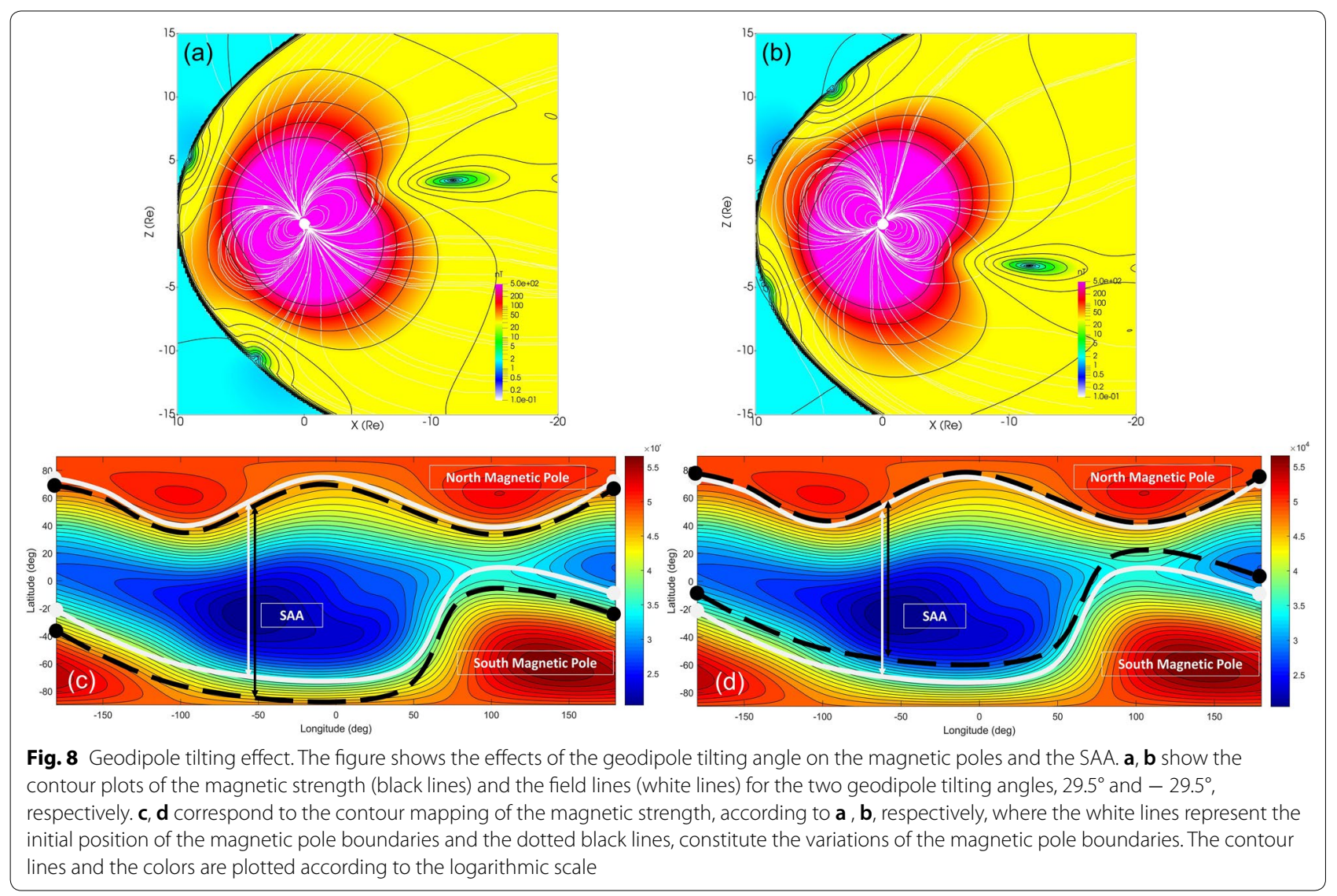

be detected visually in the projected maps, the drawn boundaries in both of the last two panels do not correspond to the calculated magnetic field variations in Panels (a) and (b), but they are actually just drawn to clarify the magnetic pole effects on the SAA area.

The SAA area and the $B_{\min } / B_{\max }$ variable can be illustrated as a "mountain" for both magnetic poles, where their height describes the magnetic field strength and their width describes the area; in addition, the "valley" describes the SAA, where its depth stands for the weak magnetic field strength and its width, the area.

From this simple concept, if one selects a positive geodipole tilting angle (Panel (a)), which means when the Earth's axis is pointing toward the sun, the distance between the north magnetic pole and the magnetopause boundary is getting smaller, so that the height of the mountain $\left(=B_{\max }\right)$ decreases and its width (= area) increases. Contrarily, the same conclusion can be derived for the south magnetic pole, where its height is increased and its area is decreased, for the same conditions. Since the variations of south the magnetic pole area are larger than the north, as shown in Fig. 7, the SAA area is getting larger, as is demonstrated in Panel (c) of Fig. 8; hence, the
SAA $B_{\min }$ is decreased. The same interpretation can also be made for the negative geodipole tilting angle case, see Panel (b) and its corresponding Panel (d).

\section{The Dst index effects on the magnetic poles and the SAA}

Figure 9 explains the Dst index effects on the magnetic poles and the SAA, which is interpreted similarly to Fig. 8. Panels (a) and (b) illustrate the contour plots of the magnetic and the field lines for the two different cases of the Dst index, $-7 \mathrm{nT}$ and $-210 \mathrm{nT}$, respectively. In Panel (b), the thick black lines correspond to the contour plot of the magnetic strength when the Dst index equals $-210 \mathrm{nT}$ and the dotted black lines are equal to $-7 \mathrm{nT}$.

From Figs.7 and 9, it can be understood that when the Dst index is decreased, the magnetotail structure is getting thinner and more extending to the night side, so that both magnetic pole areas are decreased and their $B_{\max }$ is increased. This, in turn, increases the SAA area and decreases its $B_{\min }$.

In brief, what can be concluded from this section is that the space weather parameters, the Dst index, and the geodipole tilting angle are all affecting the SAA, mainly due to the magnetic polar variations. 


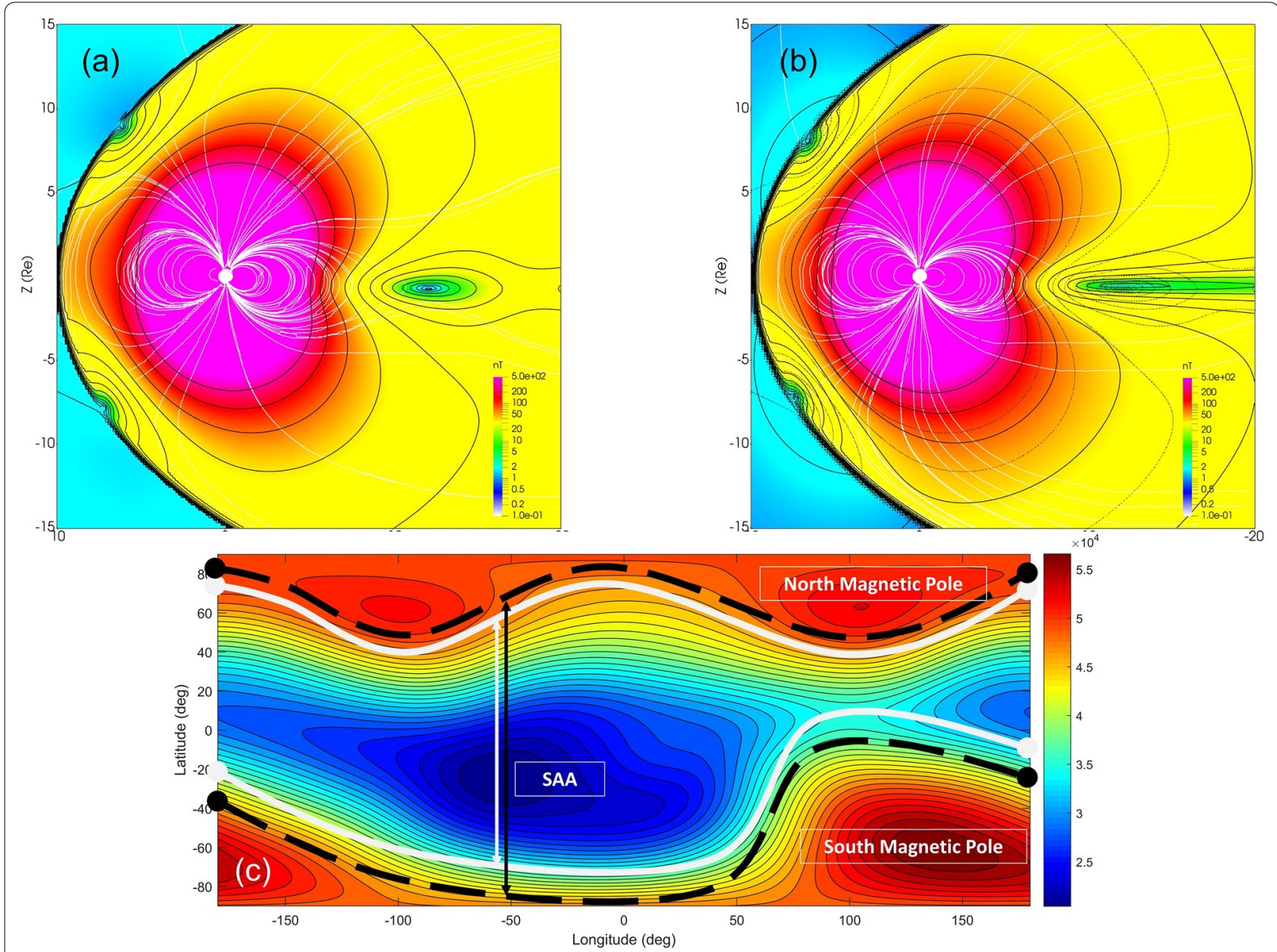

Fig. 9 Dst index effect. The figure shows the Dst index effect on the magnetic poles and on the SAA. $\mathbf{a}, \mathbf{b}$ show the contour plots of the magnetic strength (black lines) and the field lines (white lines) for the two Dst index cases, $-7 \mathrm{nT}$ (dotted lines) and - $210 \mathrm{nT}$ (solid black lines), respectively. c corresponds to the contour mapping of the magnetic strength, according to $\mathbf{b}$, where the white lines represent the initial position of the magnetic pole boundaries and the dotted black lines, the variations of the magnetic pole boundaries. Note: the contour lines and the colors are plotted according to the logarithmic scale

Several types of research have already confirmed that the $D s t$ index and the geodipole tilting angles are affecting the polar cusp and cap, such as the study of the seasonal variations and interplanetary conditions' effects on cusp regions using observations from Polar satellite (Stubbs et al. 2004), and the interpretation of the storm activity effects (Dst index) on the global magnetic field using Tsyganenko model T96 by Feshchenko et al. (2000). Moreover, Meng (1982, 1984) and Stasiewicz (1991) found that the latitudinal variations of the polar cusp zone are affected by the Dst index using observations, in addition to the size of the polar cap that was investigated by Kamide et al. (1999), as well as the diurnal variations of the polar cap and the cusp boundaries, by Sergeev (1990), besides the Polar Cap (PC) indices, that can characterize the space weather activity (Stauning et al. 2008).

\section{Diurnal variation effects on SAA response}

The analysis in the previous section was realized at a specific condition of the day, UT $=00: 00$. However, it was also interesting to investigate if the anomaly was also diurnally affected. To achieve this objective, the SAA diurnal variation of 5 January 2010 was studied, by adopting model TS05.

Figure 10 explains the variations of the SAA variables throughout a day (white area) and a night (gray shaded area), computed by implementing TS05. Even though the variations were quantitatively low, it can be detected that there is a direct correlation between the SAA variables and the day/night succession. The SAA $B_{\min }$ and the latitudinal movement of the SAA center were strongly affected and correlated with the hourly variations, where the maximum variation occurred when the SAA was facing the sun. However, the SAA area variations had 


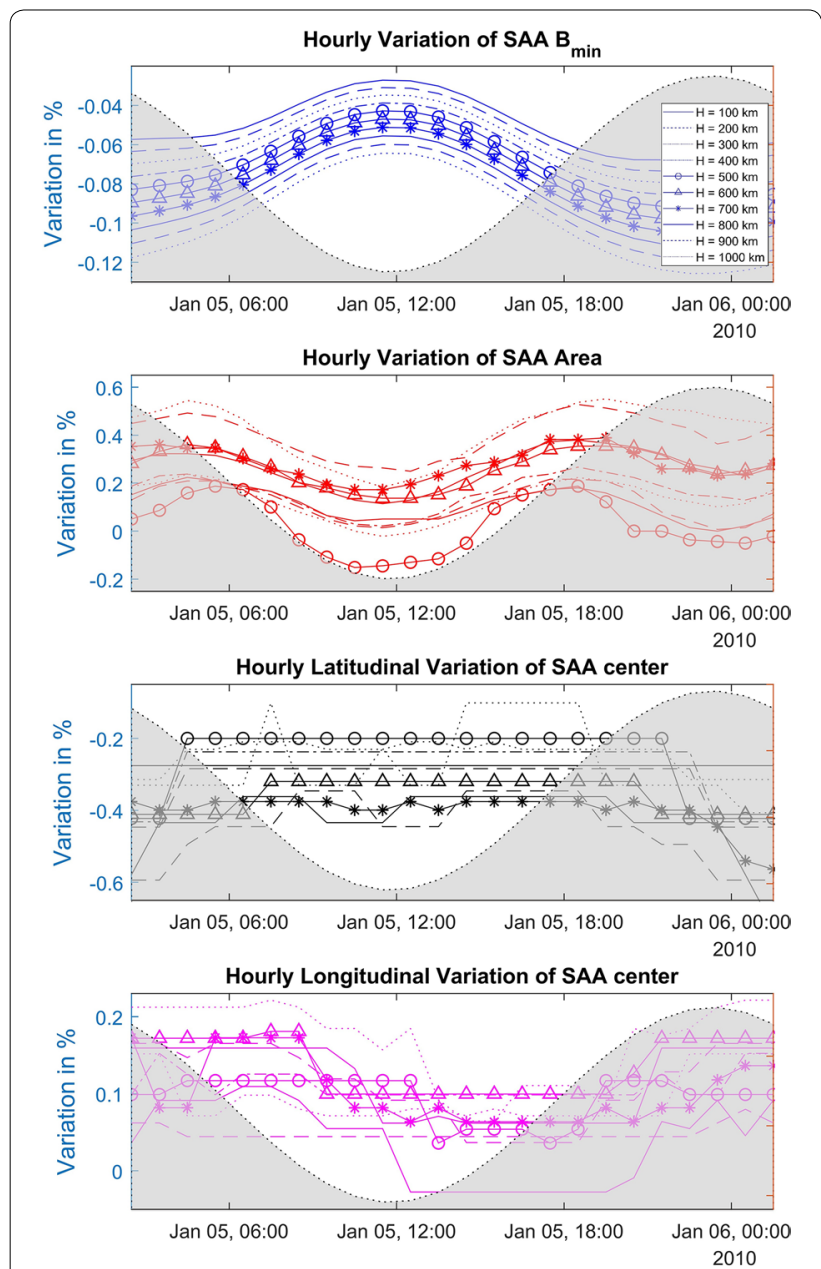

Fig. 10 SAA hourly variations. Diurnal variation of the SAA variables: the area, the $B_{\mathrm{min}}$, the latitudinal and longitudinal center movements with respect to altitude

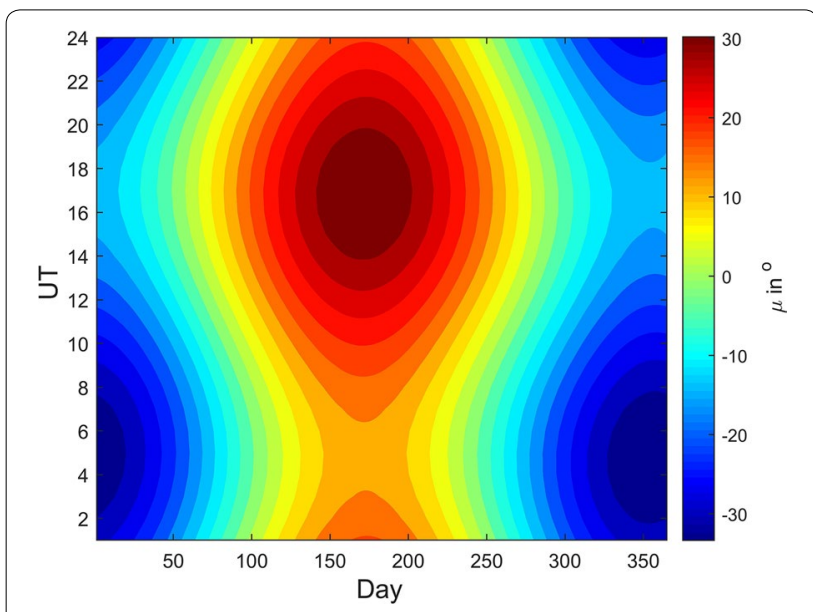

Fig. 11 Geodipole tilting angle daily and hourly variations. Daily and hourly variations of the geodipole tilting angle, computed by GEOPACK package another profile, which consisted of two minimum values, when the SAA was exactly at dayside and nightside, although the largest variations were found at daytime. The longitudinal movement of the SAA center also had another profile where a shift occurred, comparing this with the hourly succession.

Furthermore, since it was easier to interpret the variations of the SAA $B_{\min }$ and the area, the reason behind the SAA diurnal variations was better understood. It was found that when the SAA was located at nightside, the magnetotail field lines were more extended than when the SAA was located at dayside, due to the differences of the geodipole tilting angle value, (a) $\mu \approx-5^{\circ}$ at UT 15:00 and (b) $\mu \approx-25^{\circ}$ at UT 03:00, as shown in Fig. 11 . This was probably the reason why the SAA $B_{\min }$ was decreased and that the SAA area was slightly increased at nightside. Once more, the effects of the magnetotail on the SAA's response, as explained in the previous section, have been highlighted.

\section{The effects of the magnetic field threshold on the SAA area}

In this section, the effects of the magnetic field threshold in calculating the SAA area were studied. Three different values of $1.08 B_{\mathrm{min}}, 1.16 B_{\mathrm{min}}, 1.25 B_{\mathrm{min}}$ were selected, to define the SAA boundary, as plotted in Panels (a) and (c) of Fig. 12, with red, blue and green lines, respectively. Panel (a) shows the temporal variation of the SAA area, while Panel (c) shows the contour plot levels of each boundary. Panels (b) and (d) demonstrate the correlation coefficient of the SAA area, calculated based on the three different threshold values, with the Dst index, and the geodipole tilting angle $(\mu)$. It was found that each SAA area level clearly and similarly showed that both space weather parameters affected the anomaly. Moreover, it was found that as the threshold values were decreased, the SAA area was more influenced by the Dst index. Thus, the SAA area was influenced by the space weather conditions, despite the selected threshold value.

\section{A note about proton flux observations inside the SAA}

Figure 13 is another reproduction of the statistical analysis, which is a comparison between the observed data from NOAA 17 (as shown in Panels (b)) as reported by Zou et al. (2015), as well as the numerical results obtained when using Tsyganenko models T01 and TS05 (Panels (a)). The two SAA observed variables were the maximum proton flux and the corresponding flux area, whereas the other SAA variables were the SAA $B_{\min }$ and its corresponding area. It was observed that the computed results agreed well with the observations, in that the Dst index was the main space weather factor on the response of the SAA. Let one suppose that the magnetic field of the SAA is behaving like a cavity wall, where 

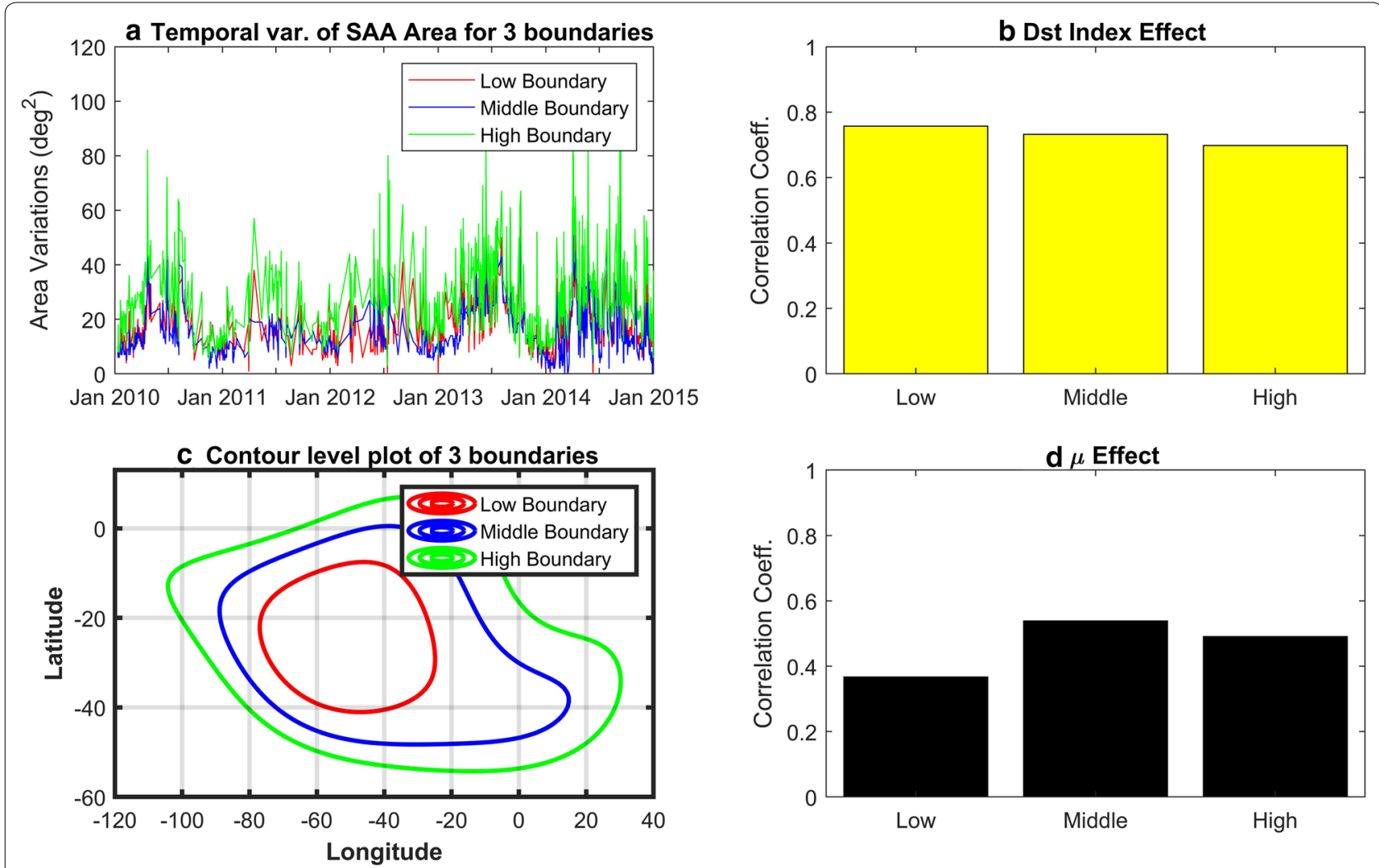

Fig. 12 Area threshold variation effect. a demonstrates the SAA area temporal variation, while $\mathbf{c}$ the contour plot level of each boundary. b, d illustrate the correlation coefficient between the SAA area, calculated based on the three different threshold values, the Dst index, and the geodipole tilting angle $(\mu)$

the precipitated protons from the inner radiation belt should move according to its variations. It had already been shown that in severe solar wind conditions, when the $D s t$ index was $<-100 \mathrm{nT}$, the SAA $B_{\min }$ is decreased and the area was increased. Thus, it is expected that both the corresponding maximum proton flux and the area should also increase. However, observations had shown the opposite of that, where the maximum protons flux and the area had been decreased. In summary, it should be noted that the proton loss, the particle energy and the time scale are considered as another important factors that could affect the proton flux inside the anomaly region, not only the magnetic field.

\section{Conclusion and summary}

The SAA magnetic field response to the space weather variations was studied by adopting Tsyganenko models T96, T01 and TS05. The SAA variables introduced in this study were the area of the SAA, the $B_{\mathrm{min}}$, and motion of the location of the SAA center. The main conclusions can be summarized as follows:
1. The Dst index and the geodipole tilting angle were the most influencing space weather parameters on the magnetic field variations of the SAA.

2. TS05, T01 and T96 enhanced the seasonal variations, namely, the Dst index, and the ram pressure on the SAA magnetic field response, respectively.

3. The dynamics of the magnetotail were considered to be an important factor of the SAA magnetic field response.

4. The magnetic field variations of the SAA were mainly driven by both magnetic poles, in a response to the space weather conditions (the Dst index and the geodipole tilting angle variations).

5. The SAA magnetic field response was also subjected to diurnal effects, where the maximum variations of $B_{\mathrm{min}}$, the area and the latitudinal movement occurred at dayside.

By recalling the main objective of the current study, the understanding of how the SAA magnetic response is influenced by the space weather parameters is considered as one of the keys that could help us to interpret the 

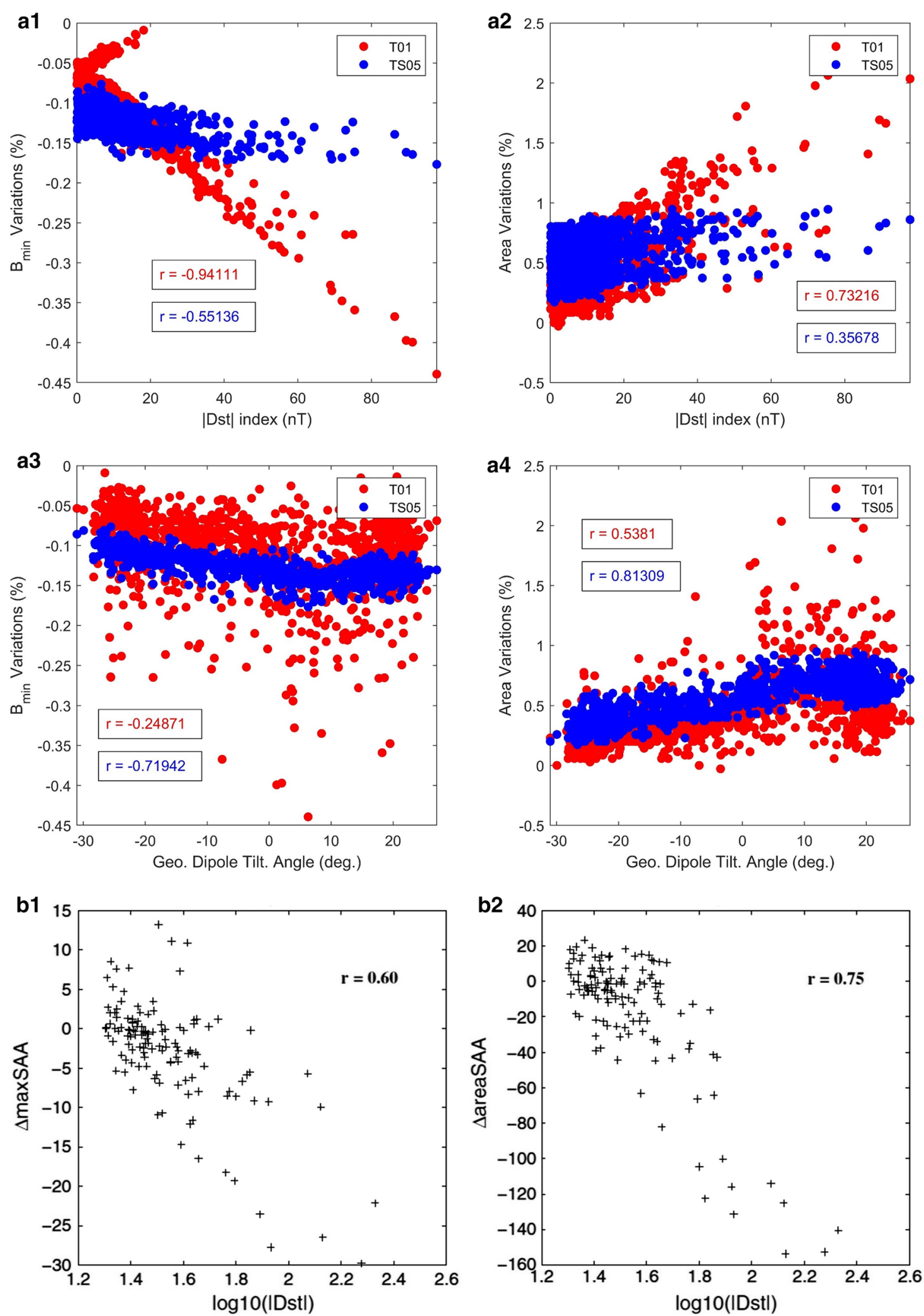

Fig. 13 Satellite observations. a represents the scatter plots of the SAA $B_{\min }$ and the area, related to the Dst index and geodipole tilting angle, as computed from Tsyganenko models T01 and TS 05 , at $800 \mathrm{~km}$. b represents the scatter plots of $\triangle$ maxSAA and $\triangle$ areaSAA, corresponding to the maximum flux values and the SAA proton flux area, respectively, with respect to the Dst index, as measured from NOAA 17 (Zou et al. 2015) 


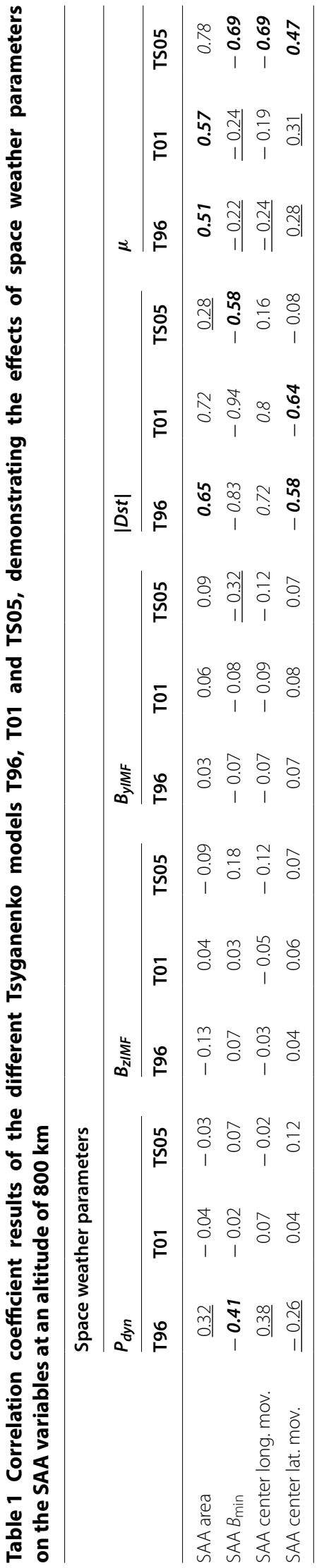


variations of the SAA proton flux response, according to the geomagnetic storms and seasonal variations, which will be carried out in a future study.

\begin{abstract}
Abbreviations
ACE: Advanced Composition Explorer (spacecraft); AE: Auroral electrojet (index); DMSP: Defense Meteorological Satellite Program (spacecraft); Dst: Disturbance storm-time (index); IB: Isotropic precipitation boundary; IGRF: International Geomagnetic Reference Field; IMF: Interplanetary magnetic field; LEO: Low earth orbit; NOAA: National Oceanic and Atmospheric Administration (spacecraft); SAA: South Atlantic Anomaly; SAMPEX: Solar Anomalous and Magnetospheric Particle Explorer (spacecraft); T79,T89,T96,T01,TS05: Tsyganenko models developed in 1979, 1989, 1996, 2001, 2005, respectively; THEMIS: Time History of Events and Macroscale Interactions during Substorms (spacecraft); UT: Universal time.
\end{abstract}

\section{Acknowledgements}

We would like to thank Prof. Shinji Saito from the Solar-Terrestrial Environment Laboratory, Nagoya University, Japan, for his exceptional support and great assistance, and also to Prof. Haruichi Washimi from the University of Alabama, USA, for his fruitful comments and suggestions.

\section{Authors' contributions}

TH and SM supervised the research project and revised the manuscript. KM developed almost the entire work. All authors read and approved the final manuscript.

\section{Funding}

Not applicable.

\section{Availability of data and materials}

The ACE solar wind and geomagnetic index data were provided from SRL, California Institute of Technology (http://www.srl.caltech.edu/ACE/) and WDC, Kyoto University (http://wdc.kugi.kyoto-u.ac.jp/dstdir/), respectively.

\section{Ethics approval and consent to participate}

Not applicable.

\section{Competing interests}

Not applicable.

\section{Author details}

${ }^{1}$ Department of Earth System Science and Technology (ESST), Interdisciplinary Graduate School of Engineering Sciences (IGSES), Kyushu University, Fukuoka, Japan. ${ }^{2}$ Aerospace Engineering Department, Faculty of Engineering, Cairo University, Giza, Egypt. ${ }^{3}$ Department of Advanced Environmental Science and Engineering, Faculty of Engineering Sciences, Kyushu University, Fukuoka, Japan. ${ }^{4}$ International Center for Space Weather Science and Education (ICSWSE), Kyushu University, Fukuoka, Japan.

\section{Received: 11 December 2019 Accepted: 24 June 2020}

Published online: 13 July 2020

\section{References}

Aubert J (2015) Geomagnetic forecasts driven by thermal wind dynamics in the Earth's core. Geophy J Int 203:1738-1751

Badhwar GD (1997) Drift rate of the South Atlantic Anomaly. J Geophys Res 102(A2):2343-2349

Casadio S, Arino O (2011) Monitoring the South Atlantic Anomaly using ATSR instrument series. Adv Space Res 48(6):1056-1066

Cottaar S, Lekic V (2016) Morphology of seismically slow lower-mantle structures. Geophy J Int 207:1122-1136

Dragt AJ (1971) Solar cycle modulation of the radiation belt proton flux. J Geophys Res 76(10):2313-2344

Feshchenko EY, Maltsev YP, Ostapenko AA (2000) Dependence of the magnetospheric magnetic field on the storm activity. In: SP-443 AWE (ed)
Congress Center of the Arctic and Antarctic Research Institute, ESA, ESA, p 431

Ganushkina NY, Pulkkinen TI, Kubyshkina MV, Sergeev VA, Lvova EA, Yahnina TA, Yahnin AG, Fritz T (2005) Proton isotropy boundaries as measured on mid- and low-altitude satellites. Annal Geophys 23:1839-1847

Girgis KM, Hada T (eds) (2018) Long-Term Variations of the Solar Wind Effects on South Atlantic Anomaly (SAA) using Tsyganenko Model, vol 4, Kyushu University, International Exchange and Innovation Conference on Engineering \& Sciences (IEICES), https://catalog.lib.kyushu-u.ac.jp/opac_detai I_md/?lang=0\&amode $=$ MD100000\&bibid $=1960662$

Grigoryan OR, Romashova WV, Petrov AN (2008) SAA drift: Experimental results. Adv Space Res 41(1):76-80

Huston S, Pfitzer K (1998) Space environment effects: low-altitude trapped radiation model. Tech. rep, Marshall Space Flight Center, NASA, Huntsville, Ala

Huston S, Kuck G, Pfitzer K (1996) Low-altitude trapped radiation model using TIROS/NOAA data. Radiat Belts Model Stand 97:1 19-122

Kamide Y, Kokubun S, Bargatze LF, Frank LA (1999) The size of the polar cap as an indicator of substorm energy. Phys Chem Earth 24(1-3):119-127

Kress BT, Hudson MK, Selesnick RS, Mertens CJ, Engel M (2015) Modeling geomagnetic cutoffs for space weather applications. J Geophy Rese Space Phys 120:5694-5702

Kronberg EA, Grigorenko EE, Haaland SE, Daly P, Delcourt DC, Luo H, Kistler LM, Dandouras I (2015) Distribution of energetic Oxygen and Hydrogen in the near-Earth plasma sheet. J Geophy Rese Space Phys 120:3415-3431. https://doi.org/10.1002/2014JA020882

Kubyshkina M, Sergeev V, Tsyganenko N, Angelopoulos V, Runov A, Singer H, Glassmeier KH, Auster HU, Baumjohann W (2009) Toward adapted timedependent magnetospheric models: A simple approach based on tuning the standard model. J Geophy Res 114(A00C21)

Malin S, Isikara A (1976) Annual variation of the geomagnetic field. Geophys J R Astron Soc 47:445-457

Meng Cl (1982) Latitudinal variation of the polar cusp during a geomagnetic storm. Geophys Res Lett 9(1):60-63

Meng Cl (1984) Dynamic variation of the auroral oval during intense magnetic storms. J Geophys Res 89(A1):227-235

Nakano G, Heckman H (1968) Evidence for solar-cycle changes in the innerbelt protons. Phys Rev Lett 20(15):806-809

Pavón-Carrasco FJ, De Santis A (2016) The South Atlantic Anomaly: the key for a possible geomagnetic reversal. Front Earth Sci 4:40

Qin M, Zhang X, Ni B, Song H, Zou H, Sun Y (2014) Solar cycle variations of trapped proton flux in the inner radiation belt. I Geophy Rese Space Phys 119:9658-9669. https://doi.org/10.1002/2014JA020300

Schaefer RK, Paxton LJ, Selby C, Ogorzalek B, Romeo G, Wolven B et al. (2016) Observation and modeling of the South Atlantic Anomaly in low earth orbit using photometric instrument data. Space Weather Int J Res Appl 14(5):330-342

Sergeev VA (1990) Polar cap and cusp boundaries at day and night. J Geomag Geoelectr 42:683-695

Sergeev VA, Tsyganenko NA (1982) Energetic particle losses and trapping boundaries as deduced from calculations with a realistic magnetic field model. Planet Space Sci 30(10):999-1006

Shevchenko IG, Sergeev V, Kubyshkina M, Angelopoulos V, Glassmeier KH, Singer HJ (2010) Estimation of magnetosphere-ionosphere mapping accuracy using isotropy boundary and themis observations. J Geophys Res115(A11206)

Shore RM, Whaler KA, Macmillan S, Beggan C,Velímský J, Olsen N, (2016) Decadal period externalmagnetic field variations determined via eigenanalysis. J GeophysRes Space Phys 121:5172-5184

Smart DF, Shea MA (1994) Geomagnetic cutoffs: a review for space dosimetry applications. Adv Space Res 14(10):787-96

Smart DF, Shea MA (2005) A review of geomagnetic cutoff rigidities for earthorbiting spacecraft. Adv Space Res 36:2012-2020

Stasiewicz K (1991) Polar cusp topology and position as a function of interplanetary magnetic field and magnetic activity: comparison of a model with Viking and other observations. J Geophys Res 96(A9):15,789-15,800

Stauning P, Troshichev O, Janzhura A (2008) The polar cap (PC) indices: relations to solar wind parameters and global magnetic activity. J Atmospheric Solar Terrest Phys 70:2246-2261

Stubbs TJ, Cargill PJ, Lockwood M, Grande M, Kellett BJ, Perry CH (2004) Extended cusp-like regions and their dependence on the polar orbit, 
seasonal variations and interplanetary conditions. J Geophys Res. https:// doi.org/10.1029/2003JA010163

Tarduno JA, Watkeys MK, Huffman TN, Cottrell RD, Blackman EG, Wendt A, Scribner CA, Wagner CL (2015) Antiquity of the South Atlantic Anomaly and evidence for top-down control on the geodynamo. Nat Commun 6:7865

Terra-Nova F, Amit H, Hartmann GA, Trindade RI, Pinheiro KJ (2017) Relating the South Atlantic Anomaly and geomagnetic flux patches. Phys Earth Planetary Interiors 266:39-53

Tsyganenko NA (1979) Subroutines and tables for calculation of the geomagnetic field. WDC-B2 issue

Tsyganenko NA (1989) A magnetospheric magnetic field model with awarped tail current sheet. Planet Space Sci 37:5-20

Tsyganenko NA (ed) (1996) Effects of the solar wind conditions in the global magnetospheric configurations as deduced from data-based field models, European Space Agency, International Conference on Substorms, Proceedings of the 3rd International Conference

Tsyganenko NA (2002a) A model of the near magnetosphere with a dawndusk asymmetry 1. Mathematical structure. J Geophy Rese Space Phys. https://doi.org/10.1029/2001JA000219\%3c/RefSource\&gt

Tsyganenko NA (2002b) A model of the near magnetosphere with a dawndusk asymmetry 2. Parameterization and fitting to observations. J Geophy Rese Space Phys. https://doi.org/10.1029/2001JA000220
Tsyganenko NA (2013) Data-based modelling of the earth's dynamic magnetosphere: a review. Annales Geophysicae 31:1745-1772

Tsyganenko NA, Sitnov MI (2005) Modeling the dynamics of the inner magnetosphere during strong geomagnetic storms. J Geophy Rese Space Phys. https://doi.org/10.1029/2004JA010798

Weiss LA, Thomsen MF, Reeves GD, McComas DJ (1997) An examination of the Tsyganenko (T89a) field model using a database of two-satellite magnetic conjunctions. J Geophy Res 102(A3):4911-4918

Ye Y, Zou H, Zong Q, Chen H, Wang Y, Yu X, Shi W (2017) The secular variation of the center of geomagnetic South Atlantic Anomaly and its effect on the distribution of inner radiation belt particles. Space Weather. https://doi. org/10.1002/2017SW001687

Zou H, Li C, Zong Q, Parks GK, Pu Z, Chen H, Xie L, Zhang X (2015) Short-term variations of the inner radiation belt in the South Atlantic Anomaly. J Geophy Rese Space Phys. https://doi.org/10.1002/2015JA021312

\section{Publisher's Note}

Springer Nature remains neutral with regard to jurisdictional claims in published maps and institutional affiliations.

\section{Submit your manuscript to a SpringerOpen ${ }^{\circ}$ journal and benefit from:}

- Convenient online submission

- Rigorous peer review

- Open access: articles freely available online

- High visibility within the field

- Retaining the copyright to your article

Submit your next manuscript at $\boldsymbol{\nabla}$ springeropen.com 